\title{
Planeación y desarrollo de proyectos como estrategia de consolidación
}

\section{Planning and project development as a consolidation strategy}

\author{
OJEDA-ESCOTO, Pedro Agustín*† \\ Universidad Tecnológica de Aguascalientes-División de Electromecánica Industrial y Energías, Blvd. Juan Pablo II 1302 \\ Exhacienda la Cantera, C.P. 20200, Ags., México
}

ID $1^{\mathrm{er}}$ Autor: Pedro Agustín, Ojeda-Escoto / ORC ID: 0000-0001-7282-4672, CVU CONACYT ID: 50027

DOI: $10.35429 / J U S D .2019 .16 .5 .1 .10$

Recibido: 30 de Junio, 2019; Aceptado 20 de Septiembre, 2019

\begin{abstract}
Resumen
Empresas dedicadas al desarrollo de proyectos comúnmente buscan aumentar su captación en base a la experiencia y por lo general dejan de lado la importancia que conlleva el fortalecimiento de la transferencia tecnológica. Aunado a esto y teniendo como necesidad extra la definición de estrategias para optimizar el progreso de los proyectos, se generó un Sistema de Gestión que ayudó a obtener un mejor control en desarrollo y definir así criterios para dar seguimiento puntual. Se establece el marco teórico tomando las bases de Planeación y Administración de Proyectos lo que permitió fundamentar dicho sistema. El enfoque utilizado para estructurarlo, permite optimizar tiempos de desarrollo y destinar un periodo de evaluación más holgado para la transferencia tecnológica del producto final. Se alcanzó la estandarización en el planteamiento, desarrollo y manufactura de los proyectos, lo que trajo como resultado, poder realizar actividades al mismo tiempo en diferentes proyectos sin presentarse desfasamiento o problemas de dependencia entre dichas actividades y, por lo tanto, la influencia estratégica para el desarrollo de cada proyecto se vio reflejada en todas las fases del proceso. Finalmente, se presenta un caso de estudio en el que fue implementado el sistema de gestión.
\end{abstract}

Proyecto, Transferencia tecnológica, Sistema de gestión

\begin{abstract}
Companies dedicated to project development commonly seek to increase their recruitment based on experience and usually leave aside the importance that entails the strengthening of technology transfer. Added to this and having as an extra need the definition of strategies to optimize the progress of the projects, was created a Management System that helped to obtain better control in development and define criteria for timely monitoring. The theoretical framework was established taking the bases of Planning and Project Management that allowed to basing this system. The approach used to structure it allows optimizing development times and assigning more comprehensive evaluation period for the technological transfer of the final product. Standardization was achieved in the design, development and manufacturing of projects, which resulted in be able to carry out activities at the same time in different projects without any offset or dependency problems between those activities and therefore, the strategic influence for the development of each project was reflected in all phases of the process. Finally, a case study is presented, in which the management system was implemented.
\end{abstract}

Project, Technology transfer, Management system

Citación: OJEDA-ESCOTO, Pedro Agustín. Planeación y desarrollo de proyectos como estrategia de consolidación. Revista del Desarrollo Urbano y Sustentable. 2019. 5-16: 1-10

\footnotetext{
*Correspondencia al autor (correo electrónico: pedro.ojeda@utags.edu.mx)

$\uparrow$ Investigador contribuyendo como primer autor
} 


\section{Introducción}

Las ideas y necesidades que detonan el planteamiento de nuevos proyectos surgen principalmente de ideas originales, necesidades internas de una empresa $u$ organización y requerimientos directos por parte de los clientes. Aunque normalmente las empresas tienen la claridad de los procesos y la manera en cómo se ejecutan, no siempre se cuenta con el soporte documental específico para cada una de las operaciones internas. La ausencia de registros y controles genera incertidumbre en los resultados y hace por consecuencia más lenta la toma de decisiones, al no contar con los datos necesarios en el momento y lugar oportuno.

La Administración de Proyectos tiene como finalidad principal la planificación, el seguimiento y control de las actividades y de los recursos humanos y materiales que intervienen en el desarrollo de un proyecto. Ya sea que se trate de un proyecto nuevo o de la ampliación y/o mejora de alguno ya existente, se muestra a continuación un esquema general de las fases involucradas en la gestión del proyecto:

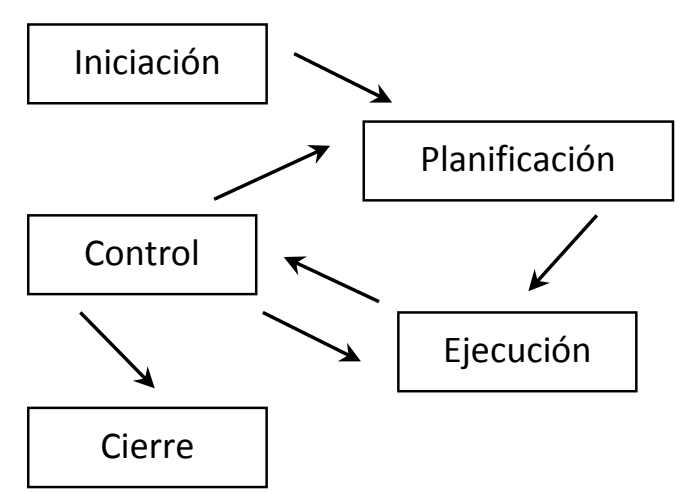

Figura 1 Esquema de Gestión de Proyectos Fuente: Elaboración Propia

\begin{tabular}{|l|l|}
\hline \multicolumn{1}{|c|}{ Fase } & \multicolumn{1}{c|}{ Descripción } \\
\hline Iniciación & Inicio del proyecto. \\
\hline Planificación & $\begin{array}{l}\text { Refinación de objetivos y selección del } \\
\text { mejor curso de acción para alcanzarlos. }\end{array}$ \\
\hline Ejecución & $\begin{array}{l}\text { Coordinación de actividades, personal y } \\
\text { recursos para llevar a cabo el plan. }\end{array}$ \\
\hline Control & $\begin{array}{l}\text { Seguimiento y medición regular del } \\
\text { progreso para identificar desviaciones del } \\
\text { plan, de modo que se puedan tomar } \\
\text { medidas correctivas cuando sea } \\
\text { necesario. }\end{array}$ \\
\hline Cierre & Formalización del cierre de proyecto. \\
\hline
\end{tabular}

Tabla 1 Indicadores del esquema de gestión de proyectos Fuente: Elaboración Propia
En la presente investigación se reporta el procedimiento para definir un Sistema de Gestión que ayudó a obtener un mejor control en el desarrollo y control de proyectos y, definir así criterios para dar seguimiento puntual. El enfoque utilizado para estructurarlo, permitió optimizar tiempos de desarrollo y destinar un periodo de evaluación más holgado para la transferencia tecnológica del producto final.

\section{Objetivo}

El objetivo principal de la presente investigación radica en mostrar un panorama general de los beneficios que se obtuvieron al implementar un Sistema de Gestión para el desarrollo, seguimiento y control de proyectos, así como también la influencia estratégica que se reflejó en todas y cada una de las fases de proceso y los resultados obtenidos en relación a la mejora en tiempos de manufactura y finalización de dichos proyectos.

\section{Marco teórico}

La investigación presentada en este artículo se fundamenta principalmente en las bases de la Planeación y Administración de Proyectos para generar e implementar un Sistema de Gestión (caso de estudio).

La gestión de proyectos como área surgida en la disciplina Administrativa, desde sus inicios se ha esforzado por la efectividad y la eficiencia al planear, organizar, dirigir y controlar los recursos asignados en una organización.

\section{Generalidades}

Hoy en día, los proyectos presentan una mayor complejidad y duración, lo que requiere de capacitación, conocimiento y desarrollo de habilidades personales de los mencionados por parte del líder, gerente o desarrollador. Igualmente, precisa de herramientas y técnicas que soporten la toma de decisiones y que permitan enfocar la atención en factores clave para lograr proyectos exitosos. Contar con una metodología para la gestión de proyectos es una estrategia organizacional para maximizar el valor de los proyectos y los resultados que estos entregan. 
Da orden y claridad al líder del proyecto, y define a cada uno de los involucrados sus responsabilidades en el desarrollo de ese proyecto, ya que una misma persona puede participar en la ejecución de varios proyectos y sus responsabilidades en cada uno de ellos pueden variar.

La metodología en la gestión de proyectos, facilita que las nuevas ideas en la organización se gestionen de la mejor manera, pues brinda elementos técnicos y estratégicos a tener en cuenta de principio a fin en la ejecución de un proyecto. Para el gerente de proyectos, e inclusive para quien no sabe del tema, la metodología representa el camino a seguir, pues esta define las fases del proyecto, mide el progreso, toma acciones correctivas basadas en fallas encontradas, ayuda a identificar y minimizar los riesgos y asigna recursos durante las diferentes fases establecidas.

La selección de una metodología implica que se seleccione el estándar de base, que se adapte a la organización y que se comunique a los miembros de la organización los beneficios que traerá utilizarla. En todos los casos, debe acompañarse este proceso con una gestión del cambio adecuada, donde con capacitación, entrenamiento y seguimiento se conozca la percepción de los empleados ante este cambio. Desde el campo de la Gestión de Proyectos, la metodología es entendida como aquella que provee una ruta para dirigir y guiar el equipo de trabajo que integra los proyectos. Igualmente, la metodología provee pautas y un método (camino) estandarizado que asegura la buena administración y promueve la calidad de los resultados.

Estas guías o pautas pueden ser una lista de cosas por hacer, un planteamiento específico, plantillas, formularios e incluso listas de verificación utilizadas durante el desarrollo de un proyecto, así como el proceso que documenta una serie de pasos y procedimientos para lograr el éxito.

La implementación de una metodología para el desarrollo de proyectos en una organización, permite materializar la estrategia organizacional por medio de herramientas que facilitan el logro de los fines propuestos desde el inicio en cada proyecto, pues involucra prácticas que optimizan y economizan recursos.

\section{Perspectivas de la Gestión de Proyectos}

Es de vital importancia pensar que los proyectos tienen un ciclo de vida con un comienzo claro y un final definido. Frecuentemente los proyectos finalizan por falta de recursos o falta de tiempo, pero los proyectos realmente habrán concluido con éxito únicamente si se logran los objetivos con el tiempo y recursos disponibles. Las etapas del ciclo de vida de un proyecto son presentadas en la Tabla 2.

Para guiar satisfactoriamente un proyecto, tiene que haber un titular encargado del mismo, es responsabilidad de dicha persona garantizar que el proyecto es viable y que se gestionará de manera imparcial el tiempo y recursos propuestos.

\begin{tabular}{|c|c|c|c|c|}
\hline $1^{\text {a }}$ Etapa & $2^{\mathrm{a}}$ Etapa & $3^{\text {a }}$ Etapa & $4^{\mathrm{a}}$ Etapa & $5^{\text {a Etapa }}$ \\
\hline $\begin{array}{l}\text { Identificació } \\
\mathrm{n} \\
\text { - se piensa } \\
\text { cuidadosame } \\
\text { nte en el } \\
\text { proyecto }\end{array}$ & $\begin{array}{l}\text { Planteamiento } \\
\text { - se planea el } \\
\text { proyecto }\end{array}$ & $\begin{array}{l}\text { Ejecución } \\
\\
\text { - se lleva a } \\
\text { cabo el } \\
\text { proyecto }\end{array}$ & 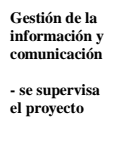 & $\begin{array}{l}\text { Conclusión } \\
\text { - el proyecto } \\
\text { finaliza }\end{array}$ \\
\hline $\begin{array}{l}\text { - Se reconoce } \\
\text { y analiza un } \\
\text { problema o } \\
\text { una } \\
\text { oportunidad } \\
\text { - se conviene } \\
\text { en el } \\
\text { propósito del } \\
\text { proyecto } \\
\text { - se examinan } \\
\text { los recursos } \\
\text { (gente, dinero } \\
\text { y tiempo) se } \\
\text { consideran } \\
\text { estrategias } \\
\text { (formas en } \\
\text { lograr el } \\
\text { propósito del } \\
\text { proyecto) }\end{array}$ & $\begin{array}{l}\text { - Se aclaran } \\
\text { los objetivos } \\
\text { - se dividen } \\
\text { las } \\
\text { estrategias } \\
\text { en una } \\
\text { secuencia de } \\
\text { actividades o } \\
\text { tareas } \\
\text { - se toman } \\
\text { decisiones } \\
\text { en cuanto a } \\
\text { quién hará } \\
\text { qué } \\
\text { - se preparan } \\
\text { presupuestos }\end{array}$ & $\begin{array}{l}\text { - comienzan } \\
\text { las } \\
\text { actividades y } \\
\text { se les } \\
\text { coordina } \\
\text { - se compila } \\
\text { información } \\
\text { sobre la } \\
\text { forma como } \\
\text { van las cosas } \\
\text { - se } \\
\text { resuelven } \\
\text { problemas } \\
\text { - se preparan } \\
\text { informes }\end{array}$ & $\begin{array}{l}\text { - Es esencial } \\
\text { la buena } \\
\text { comunicación } \\
\text { - se considera } \\
\text { la razón para } \\
\text { la } \\
\text { comunicación } \\
\text { y con quién se } \\
\text { comunica } \\
\text { - se considera } \\
\text { cómo } \\
\text { supervisar el } \\
\text { proyecto con } \\
\text { eficacia } \\
\text { - se } \\
\text { consideran } \\
\text { fuentes de } \\
\text { datos que } \\
\text { podría usarse } \\
\text { para compilar } \\
\text { información }\end{array}$ & $\begin{array}{l}\text { - Objetivos } \\
\text { cumplidos } \\
\text { - evaluación } \\
\text { realizada } \\
\text { - lecciones } \\
\text { aprendidas } \\
\text { - la } \\
\text { sustentación } \\
\text { y el impacto } \\
\text { son } \\
\text { cuestiones } \\
\text { importantes } \\
\text { - las } \\
\text { personas } \\
\text { reciben } \\
\text { reconocimie } \\
\text { nto por sus } \\
\text { logros }\end{array}$ \\
\hline
\end{tabular}

Tabla 2 Etapas del ciclo de vida de un proyecto Fuente: Elaboración propia

La gestión del proyecto implica reconocer que hay que tomar decisiones sobre el equilibrio entre lo que se puede lograr (los objetivos), el tiempo y recursos, y que la tarea continua de la gestión es procurar haya un equilibrio entre estos factores (ver Figura 2). 


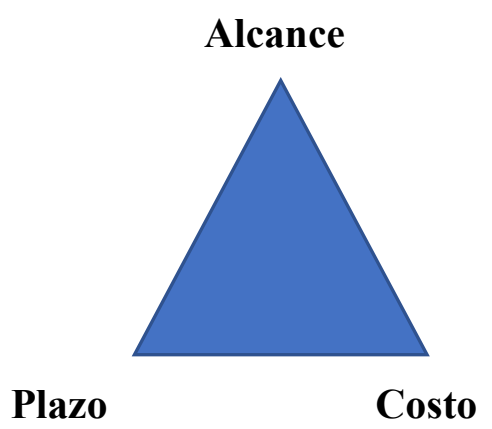

Figura 2 Factores de la estructura del proyecto Fuente: Elaboración propia

El primer factor es el alcance o resultado final de proyecto, es decir: el producto/servicio o situación que se quiere generar o a la que se busca llegar y que supone el origen y justificación del proyecto. Puede considerarse como el parámetro más importante $\mathrm{y}$ significativo, pero su consecución no es suficiente. Ha de considerarse más bien como una condición ineludible. En otras palabras: un proyecto sólo será exitoso si los recursos y el tiempo empleados han sido razonables.

El segundo factor hace referencia al costo o cantidad de recursos materiales o de personal que son necesarios para lograr el resultado esperado. En el caso de proyectos externos, el costo suele considerarse como el parámetro más importante (normalmente existe un contrato, y el proveedor deberá respetarlo o tendrá dificultades para revisar el alza del presupuesto). En proyectos internos, por el contrario, es frecuente que no sea definido de forma explícita.

Finalmente, el tercer factor es el plazo, es el objetivo que más difícilmente se cumple, convirtiéndose así en el que mejor mide el grado de calidad dentro de la gestión del proyecto. A menudo se piensa que el plazo de realización de un proyecto no debe valorarse excesivamente puesto que es algo que "casi nunca se respeta".

Los tres factores son inseparables y forman un sistema en el que la modificación de cada una de las partes afecta a las restantes. Dado que la maximización individual de los tres criterios básicos no es posible, es necesario maximizar una cierta combinación entre ellos, priorizando aquéllos que se adapten mejor a las estrategias de la empresa.
En el desarrollo de un proyecto, se tienen en general tres facetas: dimensión técnica, aplicación de conocimientos específicos de cada área de trabajo, esto es, cumplir con una forma de trabajar y requisitos (Know-How) que cada área impone; dimensión humana, un proyecto es un complejo entramado de relaciones profesionales donde conviven un gran número de intereses a veces contrapuestos; variable "gestión", se hace referencia a algo que a veces se menosprecia porque no es tan espectacular o visible como otros elementos pero que es el catalizador que permite que el resto de los elementos se comporten adecuadamente. Haciendo una conjunción de las facetas mencionadas anteriormente, en la Figura 3 se presenta la visión general de dicho esquema.

Establecer los procedimientos para la toma de decisiones también contribuye en gran medida al éxito del proyecto. Establecer un procedimiento para la resolución de problemas tiene un impacto similar. Registrar sistemáticamente la evolución de cada uno de los temas tratados permite identificar aquéllos en los que es necesario centrarse identificando su impacto potencial. Los problemas que no pueden ser resueltos de forma inmediata deben ser asignados como una actividad "especial" para que se resuelvan o, por lo menos, se aborden posibles soluciones.

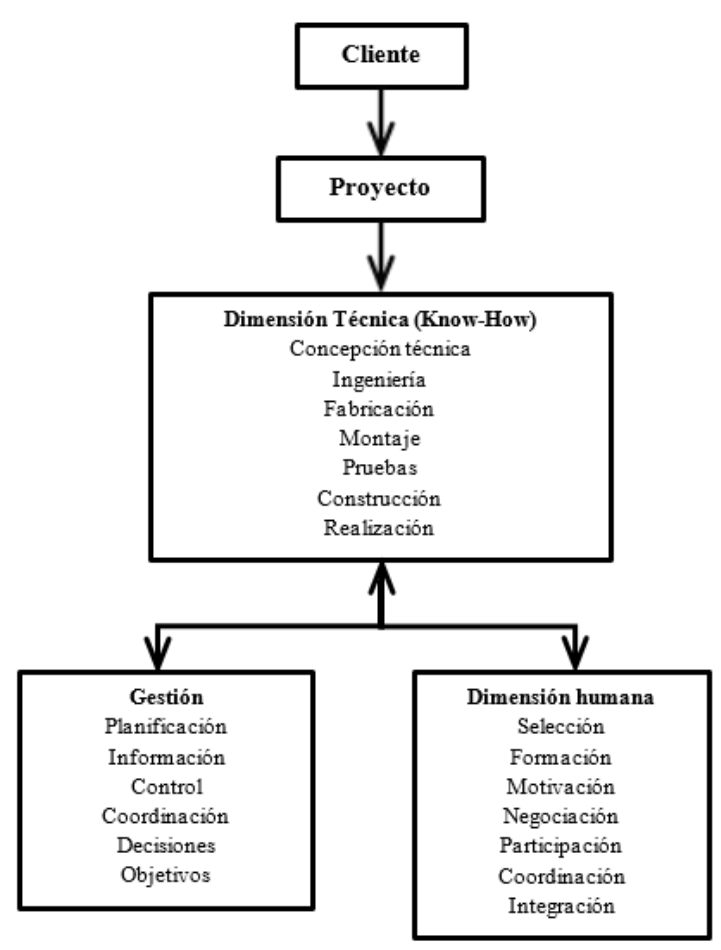

Figura 3 Facetas de un proyecto

Fuente: Elaboración propia 
Por otro lado, si se habla específicamente de establecer una visión estratégica, se definen 3 tipos de planeación, que facilitan el contar con una visión que va de lo general a lo particular, tomando en cuenta, primero las estrategias ligadas directamente de la Visión y Misión Institucionales, después la planeación de las tácticas o metodologías específicas que se van a utilizar para lograrlo, y por último definir las acciones concretas o planes que ilustren más específicamente las acciones que se han de llevar a cabo para cumplir con las metas de un área y que estén relacionadas con la forma en que la organización ha definido alcanzar sus objetivos Institucionales.

La planeación estratégica es sistemática, es decir, es organizada y conducida con base en una realidad entendida, es un proceso que se inicia con el establecimiento de metas organizacionales, define metodología, prioridades y políticas para lograr metas específicas; desarrolla planes detallados para asegurar la implantación de las estrategias y obtener así los fines buscados. Es un proceso para decidir de antemano el tipo de esfuerzo de implantación que se debe hacer, cuándo y cómo se debe realizar, quién lo llevará a cabo y qué se hará con los resultados.

La planeación táctica es aplicable cuando se cuenta con una visión a futuro que va definiendo el camino y se han establecido los objetivos que deberán ser alcanzados, es necesario entonces: contar con un plan de acción para determinar las actividades, los medios y el orden en que serán implementados, pues es así como se establece la "táctica" o método para alcanzar dichos objetivos; definir los pasos, las prioridades a seguir, las responsabilidades y hasta las personas que se encargarán de cumplir las metas previamente señaladas.

Dentro de todas las áreas de la organización, se tiene que llevar a cabo una planeación más detallada. Cada departamento funcional (mercadotecnia, finanzas, contaduría, compras, producción, personal y otros) desempeña un papel importante dentro del proceso de planeación estratégica. A los niveles gerenciales y directivos les corresponde, tanto el diseño como la implementación de las tácticas a utilizar, para alcanzar los objetivos.
Para el caso de una planeación operativa, se refiere a las actividades que se realizan de manera cotidiana, para mantener el ritmo de la operación del área correspondiente. Tiene su enfoque en el presente, es en donde se desarrollan las actividades que conforman el cumplimiento de metas que en su conjunto van a lograr los objetivos propuestos. Se diferencia de la planeación táctica en que la primera (la planeación táctica), se refiere a la puesta en marcha y la segunda a la manera en que se pretende llevar a cabo la operación.

\section{Análisis y Resultados}

\section{Caso de estudio}

El caso de estudio referido en el presente artículo se trata de un Sistema de Gestión que fue implementado en un centro de desarrollo de ingeniería. Dicho centro es una empresa $100 \%$ mexicana dedicada a ofrecer soluciones tecnológicas con las siguientes capacidades: diseño mecánico, diseño industrial, automatización, pruebas y validación, análisis por elemento finito, prototipos rápidos, entre otros.

\section{Planteamiento}

Se realizó una revisión de todos los proyectos desarrollados en un Centro de Investigación y Desarrollo para ubicar puntos clave $\mathrm{y}$ situaciones que ayudaran a definir las estrategias de optimización.

La principal necesidad que se tenía era el poder definir estrategias para optimizar el desarrollo de un proyecto, sobre todo para desarrollar una cantidad considerable de proyectos al mismo tiempo (por lo menos, más de 5 proyectos a la vez) y poder puntualizar y optimizar los tiempos y recursos destinados al desarrollo y manufactura en cada uno de ellos. Entonces, se definió como objetivo principal el poder tener un sistema que ayude a tener un mejor control en el desarrollo de cada uno de los proyectos y definir así los criterios para poder dar un seguimiento puntual desde el diseño hasta la manufactura y pruebas de funcionamiento. 


\section{Método de Investigación y definición del Sistema de Gestión}

Para el desarrollo de la propuesta de Sistema de Gestión se tomó de base los resultados obtenidos en el desarrollo de los proyectos ya finalizados del Centro de Investigación. Una vez realizado dicho análisis de información, se decidió conjuntar dos entes, por un lado, el modelo de los procedimientos operativos que gobiernan la organización y por el otro, el esquema propuesto para el desarrollo de todos y cada uno de los nuevos proyectos.

\section{Modelo de procedimientos operativos}

El modelo bajo el cual trabaja la organización es basado en la disciplina conocida como Gemba-Kaizen, en donde Gemba significa lugar de trabajo y Kaizen se refiere a la utilización de herramientas del sentido común y de bajo costo, está enfocado en el control de la operación bajo 5Ms: (Materiales, Maquinaria, Mano de Obra, Método y Medio Ambiente); con ello se ha pretendido estandarizar el enfoque de pensamiento de todo el personal de la organización, desde el Gerente General hasta el empleado de menor rango de responsabilidad en dicha organización (ver figura 4).

\section{Esquema para desarrollo de nuevos proyectos}

Como se comentó anteriormente, fue realizado un análisis de todos los proyectos finalizados en el centro de investigación. En cada uno de ellos se revisaron los objetivos, procesos y recursos utilizados para su administración. En el esquema de la figura 5 se muestra la propuesta para dar guía al desarrollo de cada uno de los nuevos proyectos que son captados por el centro de investigación.

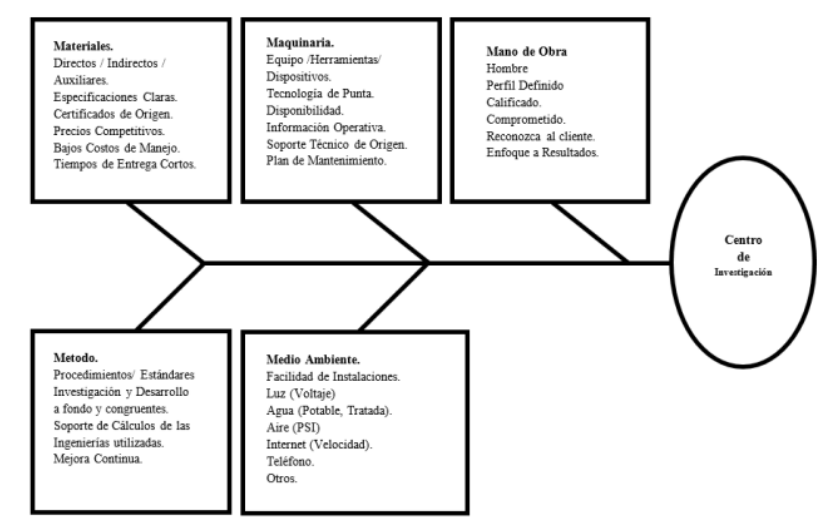

Figura 4 Modelo de Enfoque de la Organización Fuente: Elaboración Propia

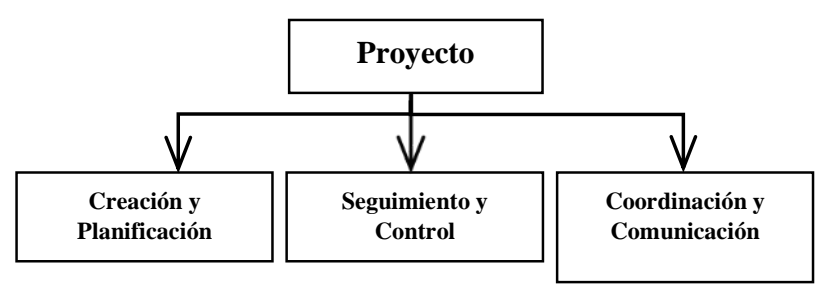

Figura 5 Esquema de proyecto Fuente: Elaboración Propia

A continuación, se presenta de una manera sintetizada (por cuestiones de confidencialidad) los puntos importantes e involucrados en cada una de las tres fases definidas:

\section{Creación y Planificación (ver figura 6)}

Especificaciones del proyecto.

- Descripción, objetivos y alcances.

- $\quad$ Fecha de ejecución y finalización.

- $\quad$ Entregable final.

Definición del esquema del proyecto.

- $\quad$ Fases del proyecto.

- $\quad$ Actividades asignadas a cada fase.

Definición del calendario del proyecto.

- Horarios reales de actividades.

Determinación de las características de cada actividad.

- Definición de características de actividad.

Tiempo definido por actividad.

Localización de puntos claves de control.

- Habilitado de material.

Búsqueda de dependencias entre actividades.

- Determinación de dependencia entre actividades:

- Fin - Inicio: el inicio de la actividad dependiente está ligado a la finalización de la precedente.

- $\quad$ Inicio - Inicio: el inicio de la actividad dependiente está ligado al inicio de la precedente.

Fin - Fin: para poder completar la actividad dependiente se debe finalizar la precedente.

Revisión y análisis crítico del proyecto.

- Verificación de tiempos asignados a cada actividad.

- Seguimiento de dependencia entre actividades. 
- Consecuencia por retraso de una actividad.

Incumplimiento de avance (Sanciones).

*Conflicto en programación de actividad.

**Conflicto en proveeduría de material.

\section{Seguimiento y Control}

Creación de plan de referencia.

- Seguimiento a la planificación del proyecto.

- $\quad$ Seguimiento del desarrollo del proyecto.

Recolección de datos reales (avances)

- Determinar periodicidad del seguimiento semanal, quincenal, mensual, en función del periodo de duración de la actividad).

Determinar información a recolectar por actividad (Fechas de inicio y finalización real, tiempo faltante para completar actividad iniciada, recursos utilizados y por utilizar).

Seguimiento de plan original.

- Actualización de programación con información real para obtención de estado real del proyecto.

- Seguimiento a proveeduría de material.

- Determinar ajustes que se podrían utilizar para corregir desviaciones en la programación.

\section{Coordinación y Comunicación}

Informe general

- Resultados obtenidos en el desarrollo del proyecto.

- $\quad$ Puntos de control definidos.

- $\quad$ Recursos necesarios y destinados para la correcta ejecución del proyecto.

Costos reales.

Informe local.

- Organización de actividades.

- Flujo de trabajo e información por actividad.

- $\quad$ Fecha de inicio y avance por actividad.

- $\quad$ Recursos implicados en cada actividad.

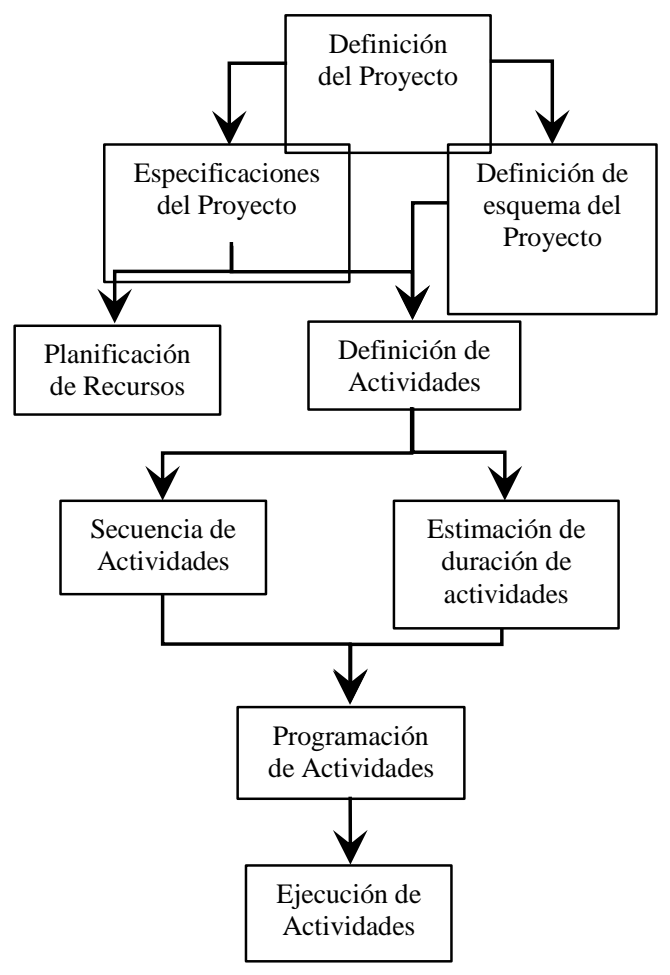

Figura 6 Etapa de creación y planificación Fuente: Elaboración Propia

\section{Resultados y discusión}

Se desarrolló un Sistema de Gestión conformado por una serie de formatos, los cuales se presentaron y revisaron en conjunto con la Dirección General y la Gerencia de Operaciones para aprobarlos definitivamente e iniciar con la implementación en todos y cada uno de los proyectos en desarrollo.

En la Figura 7 se muestra el mapeo de proceso y los documentos generados y, en la Figura 8 se muestra el mapeo de los documentos generados para complemento de dicho sistema de gestión.

El sistema desarrollado aporta criterios para poder optimizar los tiempos en el desarrollo de un proyecto. Se alcanzó la estandarización en el planteamiento, desarrollo y manufactura de todos y cada uno de los proyectos, lo que trajo como resultado, la realización de actividades al mismo tiempo en proyectos diferentes sin presentarse algún desfasamiento o problemas de dependencia entre dichas actividades.

La influencia estratégica para el desarrollo de cada proyecto se vio reflejada en todas y cada una de las fases del proceso, optimizando así el tiempo destinado a la transferencia tecnológica del producto desarrollado. proyectos como estrategia de consolidación. Revista del Desarrollo Urbano y Sustentable. 2019. 
En el momento de la implementación del Sistema de Gestión se encontraban en desarrollo 7 proyectos y fue posible tener un buen control y seguimiento en cada uno de ellos, optimizándose los resultados y tiempos de Manufactura hasta en un $40 \%$ menos con respecto a los proyectos realizados en años anteriores.

Procedimientos Generales 7

\begin{tabular}{|c|c|c|c|}
\hline $\begin{array}{l}\text { Solicitud } \\
\text { Recepcion y } \\
\text { Generacion }\end{array}$ & $\begin{array}{l}\text { Diseño: } \\
\text { Investigacion y } \\
\text { Desarrollo }\end{array}$ & Compras & $\begin{array}{l}\text { Recibo de } \\
\text { Materiales }\end{array}$ \\
\hline Fabricacion & $\begin{array}{l}\text { Ensamble } \\
\text { Pruebas }\end{array}$ & $\begin{array}{l}\text { Transferencia } \\
\text { Tecnologica }\end{array}$ & \\
\hline
\end{tabular}

Registros De Procedimientos Generales 21

\begin{tabular}{|l|l|l|l|}
\hline CIDA-SOL-01 & CIDA-DIS-01 & CIDA-COM-03 & CIDA-RMT-01 \\
CIDA-REC-01 & CIDA-DES-01 & CIDA-COM-04 & CIDA-RMT-02 \\
CIDA-REC-02 & CIDA-DES-02 & & \\
CIDA-GEN-01 & CIDA-DES-03 & & \\
CIDA-PRO-01 & CIDA-COM-01 & & \\
\hline
\end{tabular}

\begin{tabular}{|c|c|c|}
\hline CIDA-EVA-01 & CIDA-EVA-01 & CIDA-TRT-01 \\
& & CIDA-GAR-01 \\
& & CIDA-MOP-01 \\
& & CIDA-MTO-01 \\
\hline
\end{tabular}

Procedimientos Administrativos 3

\begin{tabular}{|c|l|l|}
\hline $\begin{array}{c}\text { Politica de } \\
\text { Compras } \\
\text { Interplantas }\end{array}$ & $\begin{array}{l}\text { Tranf. de } \\
\text { Materiales } \\
\text { Interplantas }\end{array}$ & $\begin{array}{l}\text { Tranf. de } \\
\text { Manufactura } \\
\text { Interplantas }\end{array}$ \\
\hline
\end{tabular}

Registros de Procedimientos Administrativos 7

\begin{tabular}{|l|l|l|}
\hline CIDA-COM-05 & CIDA-TMI-01 & CIDA-TMF-01 \\
& CIDA-TMI-02 \\
& CIDA-TMI-03 & CIDA-TMF-02 \\
CIDA-TMF-03 \\
\hline
\end{tabular}

Total de Procedimientos 10

Total de Registros $\quad 28$

Figura 7 Mapeo de procesos y generación de documentos del sistema de gestión

Fuente: Elaboración Propia

Es importante comentar también que la captación de proyectos del Centro de Investigación y Desarrollo ha aumentado considerablemente y se espera que para los próximos años se incremente entre un $30 \%$ y $45 \%$ más que el récord actual.

En los Gráficos 1, 2 y 3 se muestran los comparativos del impacto que se ha tenido desde la implementación de dicho sistema.

\section{Procedimientos Generales 7}

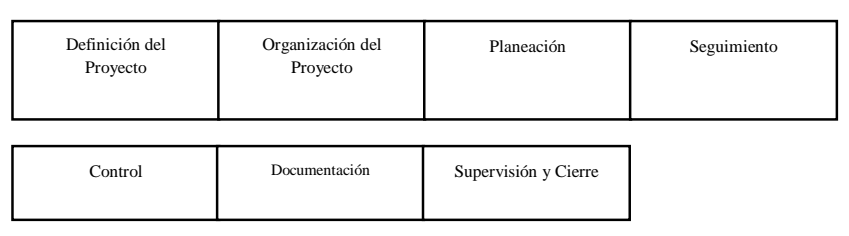

Registros de Procedimientos Generales 24

\begin{tabular}{|c|c|c|c|}
\hline $\begin{array}{l}\text { FORM01-GP-01 } \\
\text { FORM02-RP-01 }\end{array}$ & $\begin{array}{l}\text { FORM03-EP-01 } \\
\text { FORM04-MR-01 }\end{array}$ & $\begin{array}{l}\text { FORM05-CA-01 } \\
\text { FORM05A-CA-01 } \\
\text { FORM05B-SCA-01 } \\
\text { FORM06-MV-01 }\end{array}$ & $\begin{array}{l}\text { FORM07A-SCA-01 } \\
\text { FORM07B-SCA-01 } \\
\text { FORM07C-SCA-01 } \\
\text { FORM07D-SCA-01 } \\
\text { FORM07E-SCA-01 } \\
\text { FORM07F-SCA-01 }\end{array}$ \\
\hline $\begin{array}{l}\text { FORM08A-EAA-01 } \\
\text { FORM08B-EA-01 } \\
\text { FORM08C-EAA-01 } \\
\text { FORM08-EA-EA-01 } \\
\text { FORM08E-EAA-01 } \\
\text { FORM08F-EAA-01 }\end{array}$ & $\begin{array}{l}\text { FORM09-FR-01 } \\
\text { FORM10-AC-01 }\end{array}$ & $\begin{array}{l}\text { FORM11-SF-01 } \\
\text { FORM12-CP-01 }\end{array}$ & \\
\hline
\end{tabular}

Figura 8 Mapeo de documentos complementarios del sistema de gestión

Fuente: Elaboración Propia

6 Desarrollo de Proyectos

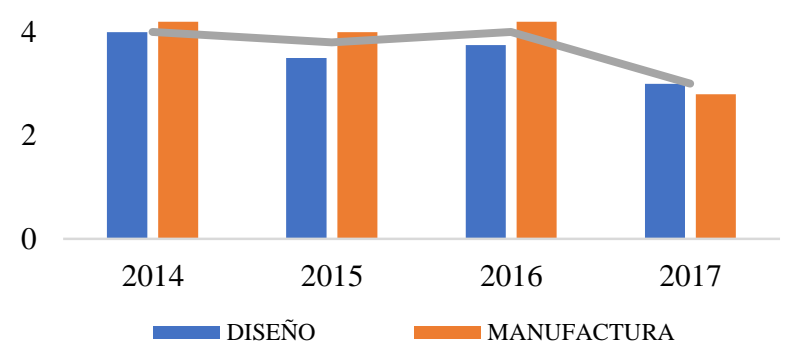

Gráfico 1 Desarrollo de Proyectos Fuente: Elaboración Propia

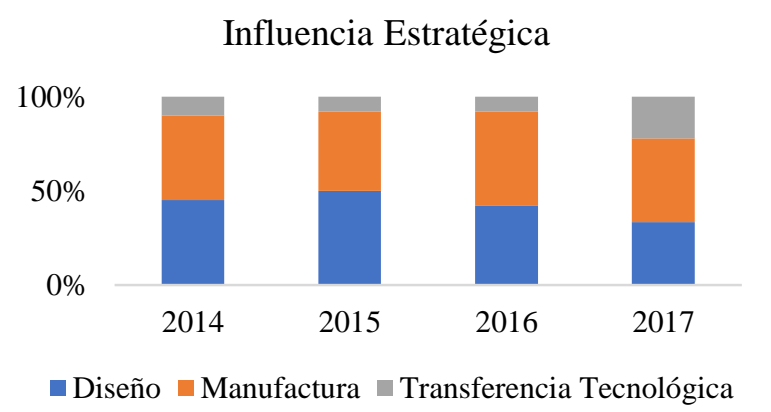

Gráfico 2 Influencia Estratégica

Fuente: Elaboración Propia

Captación de Proyectos

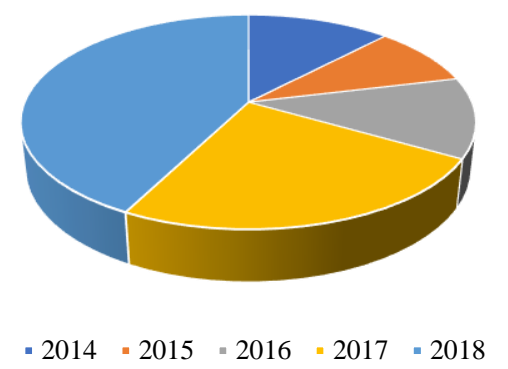

Gráfico 3 Captación de Proyectos

Fuente: Elaboración Propia

OJEDA-ESCOTO, Pedro Agustín. Planeación y desarrollo de proyectos como estrategia de consolidación. Revista del Desarrollo Urbano y Sustentable. 2019. 
Algunas ventajas competitivas que se generaron con el desarrollo del sistema de gestión en el centro de investigación son:

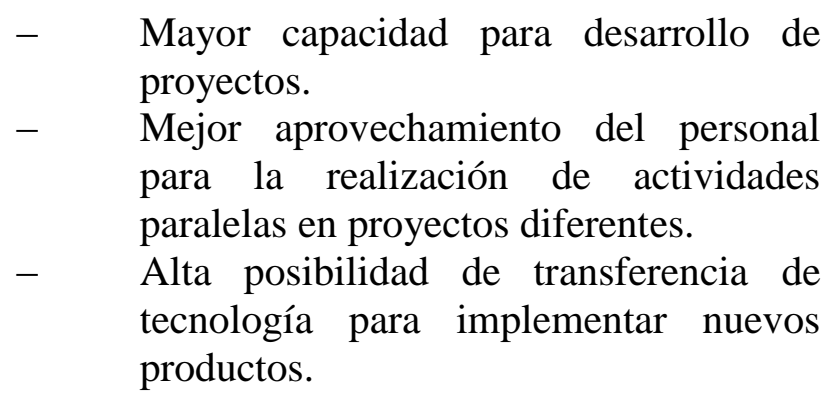

\section{Síntesis de impacto}

Se desarrolló una Sistema de Gestión que optimiza los tiempos de desarrollo de un proyecto $y$ se utiliza un proceso de investigación que hace más productivo el seguimiento de las actividades realizadas por el personal.

El sistema desarrollado se ha implementado y compartido internamente en el Centro de Investigación y Desarrollo para su uso en los actuales y futuros proyectos. Así mismo, se estimuló el uso del sistema para desarrollar e implementar mejoras en otras áreas del Centro, entre ellas la de Administración y Manufactura.

Los resultados de la práctica han ayudado a generar un mejor ambiente laboral entre todos los empleados aumentando la comunicación entre la Dirección General, la Gerencia de Operaciones y el Departamento de Diseño e Ingeniería. Además, es posible trabajar de una forma más organizada entre las diferentes áreas del Centro.

\section{Agradecimiento}

El autor del presente artículo desea agradecer el apoyo técnico, económico y logístico de la División de Electromecánica Industrial y Energías de la Universidad Tecnológica de Aguascalientes.

\section{Conclusiones}

Con el desarrollo e implementación del "Sistema de Gestión, Seguimiento y Control de Proyectos" se aportó base tecnológica para la optimización de tiempos y mejora del uso de recursos en el desarrollo de un proyecto.
Se obtuvo un mejor control de cada uno de ellos y fue posible dar un seguimiento efectivo desde el inicio del diseño hasta la manufactura y pruebas de funcionamiento.

Al optimizarse dichos tiempos en el desarrollo de los proyectos fue posible destinar un periodo de evaluación para la transferencia de la tecnología desarrollada a un producto funcional para las etapas de producción y distribución.

Finalmente, es importante comentar que con el desarrollo del sistema de gestión se logró reforzar el trabajo en equipo dentro del centro de investigación; puesto que, con el uso del sistema se desarrollan los proyectos en un menor tiempo, impactando directamente también en los tiempos de fabricación.

\section{Referencias}

Aken, V. J. (2004). Management research based on the paradigm of the design sciences: the quest for field-tested and grounded technological rules. Journal of Management Studies, (41), 219-246.

Baca, G. (2013). Evaluación de proyectos. México: McGraw-Hill.

Charvat, J. (2003). Project management methodologies. Selecting, Implementing, and Supporting Methodologies and Processes for Projects. New Jersey: John Wiley \& Sons.

Dennehy D., Adam, F, \& Carton, F. (2016). Leveraging design thinking to innovate. Open Innovation: A Multifaceted Perspective Part 1. Singapore: World Scientific Publishing Co., 159-179.

Dorst, K. (2006). Design problems and design paradoxes. Design issues, (22), 4-14.

Dorst, K. (2011). The core of design thinking and its application. Design Studies (32), 521532.

Fortune, J., White, D., Jugdev, K. \&Walker, D. (2011). Looking again at current practice in project management. International Journal of Managing Projects in Business, (4), 553-572. 
Gray, C. F. \& Larson, E. W. (2009). Administración de proyectos. México: McGraw-Hill.

Ika, L. A., Diallo, A. \& Thuillier, D. (2010). Project Management in the international development industry: The project coordinator's perspective. International Journal of Managing Projects in Business, (3), 61-93.

Ingle, B. (2013). Design Thinking for Entrepreneurs and Small Businesses: Putting the Power of Design to Work. Berkeley, CA: Apress.

Kerzner, H. (1989). Project Management. Third Edition, New York: Van Nostrand Reinhold.

Kimbell, L. (2011). Rethinking design thinking. Design and Culture, (3), 285-305.

Luchs, M. (2016). A brief introduction to design thinking. Design thinking: new product development essentials from the PDMA, 1-12.

Montes-Guerra, M. I., Gimena R. F. \& DíezSilva, H. M. (2015). Estándares y metodologías: Instrumentos esenciales para la aplicación de la dirección de proyectos. Revista de Tecnología, (12), 11-23.

Montes-Guerra, M. I., Gimena, R. F., PérezEzcurdia, M. A. \& Díez-Silva, H. M. (2011). Exploring Project Management Bodies of Knowledge and Monitoring \& Control Orientation. In XV International Congress on Project Engineering (Ed.).

Shenhar, A. J. \& Dvir, D. (2007). Project Management Research: The Challenge and Opportunity. Project Management Journal, (38), 93-99.

Whitbeck, C. (1998). Ethics in engineering practice and research. Cambridge UK: Cambridge University Press.

White, D. \& Fortune, J. (2002). Current practice in project management - an empirical study. International Journal of Project Management, (20), 1-11. 